\title{
Role of RNA-interference-induced zinc finger protein 139 suppression in gastric cancer cell sensitivity to chemotherapeutic agents
}

\author{
YING-JIE HAO, YONG LI, LI-QIAO FAN, QUN ZHAO, BI-BO TAN, ZHI-KAI JIAO, \\ XUE-FENG ZHAO, ZHI-DONG ZHANG and DONG WANG \\ Third Department of General Surgery, The Fourth Hospital of Hebei Medical University, \\ Shijiazhuang, Hebei 050011, P.R. China
}

Received July 16, 2014; Accepted June 3, 2015

DOI: $10.3892 / 01.2015 .3421$

\begin{abstract}
Zinc finger proteins (ZNFs) are a class of proteins widely distributed in the human genome, which have been found to play a role in the regulation of gene transcription and the occurrence and development of gastric cancer (GC). ZNF139 was found to be associated with GC in our previous experiments. The present study aimed to analyse the differences in ZNF139 protein expression in SGC7901 GC cells and in situ grafted GC tumors in nude mice prior to and following RNA interference inhibition, and to investigate the mechanisms underlying ZNF139 involvement in the occurrence, development and chemosensitivity of GC. A ZNF139-targeted small interfering (si)RNA plasmid was constructed and transfected into the cancer cells and in situ grafted tumors. The MTT assay was used to investigate the alterations in chemosensitivity prior to and following transfection of siRNA-ZNF139. The two-dimensional difference gel electrophoresis and liquid chromatography-mass spectrometry techniques were used to identify the different protein points prior to and following siRNA-ZNF139 transfection. Western blot analysis was performed to confirm the identified proteins. In the siRNA-ZNF139 group, the growth of the cancer cells and in situ grafted tumors significantly decreased. However, the post-interference chemosensitivity to 5-fluorouracil, cisplatin and mitomycin $\mathrm{C}$ significantly increased. In the in vivo and in vitro experiments, the expression of pyridoxal kinase (PDXK) was upregulated, whereas the expression levels of annexin A2 (ANXA2) and fascin were downregulated following transfection. Western blot analysis confirmed the results for PDXK, ANXA2 and fascin by proteomics. Therefore, ZNF139 may
\end{abstract}

Correspondence to: Professor Yong Li, Third Department of General Surgery, The Fourth Hospital of Hebei Medical University, 12 Jiankang Road, Qiaodong, Shijiazhuang, Hebei 050011, P.R. China

E-mail: yonglicn@126.com

Key words: gastric cancer orthotopic transplantation, SGC7901 gastric cancer cells, chemotherapeutic sensitivity, proteomics, RNA interference, zinc finger protein 139 participate in the occurrence, development and chemosensitivity of GC by promoting the expression of ANXA 2 and fascin, while inhibiting the expression of PDXK.

\section{Introduction}

Gastric cancer (GC) is a major cause of morbidity and mortality and has become a major public health concern worldwide (1). The early symptoms of GC are atypical; therefore, when patients develop apparent symptoms, the disease has already progressed to an advanced stage. Therefore, the opportunity for radical surgery for these patients is missed, and chemotherapy becomes the primary means of treatment.

RNA interference (RNAi) is the phenomenon of post-transcriptional gene silencing caused by double-stranded (ds) RNA (2). RNAi is considered a revolutionary novel post-gene transcriptional regulation technology due to its unique efficiency and specificity. This technology has become a powerful tool for the study of gene function and has provided novel techniques and application prospects for specific gene therapy. The RNAi technology is becoming known for gene function and regulation analysis in the post-genomic era.

Zinc finger proteins (ZNFs) are a class of proteins widely distributed in the human genome. ZNFs have been shown to regulate gene transcription, thus playing an important role in the occurrence and development of GC (3). It was previously demonstrated that the ZNF139 gene is closely associated with GC (4).

Proteomics relies on the bilateral fluorescence two-dimentional difference gel electrophoresis (2D-DIGE) to separate different proteins, which are then identified and analysed by mass spectrometry and bioinformatics (5). Proteomics technology is currently being used in various fields of biology due to its high sensitivity, accuracy and throughput. A previous study demonstrated that the ZNF139 gene is closely associated with GC (4). The aim of the present study was to further investigate the mechanisms underlying the role of ZNF139 in the occurrence, development and chemosensitivity of GC.

Based on the principle of RNAi, a ZNF139-targeted small interfering (si)RNA plasmid was developed in this study. The different proteins prior to and following siRNA-ZNF139 transfection were analyzed to provide a theoretical basis for 
A

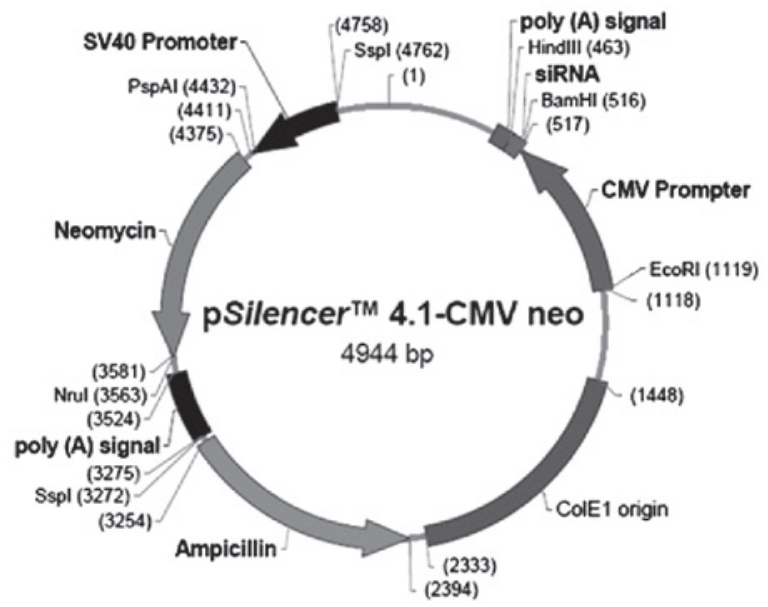

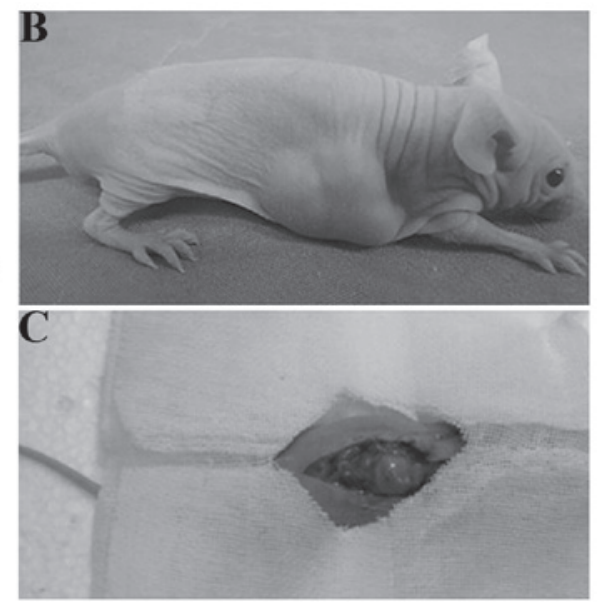

Figure 1. Construction and transplantation. (A) Plasmid carrying the small interfering (si)RNA. (B) Subcutaneous tumor transplant. (C) Orthotopic tumor transplant.

the mechanisms underlying the role of ZNF139 in the occurrence, development and chemosensitivity of GC.

\section{Materials and methods}

Construction of siRNA-ZNF139. The 19-bp siRNA and ineffective interference control fragment were designed by the siRNA online software (http://i.cs.hku.hk/ sirna/software/ sirna.php). The siRNA-ZNF139 template sequences were 5'-ACCTCGGAAGATTCAGCAT-3' (sense strand) and 5'-ATGCTGAATCTTCCGAGGT-3' (antisense strand). The ineffective interference control sequences were 5'-GACGAGTTGACTGCGATTG-3' (sense strand) and 5'-CAATCGCAGTCAACTCGTC-3' (antisense strand). The pSilencerTM 4.1-CMV neo-ZNF139 siRNA plasmid (Shanghai GenePharma Co., Ltd., Shanghai, China; Fig. 1A) and Escherichia coli were used to construct the engineered bacteria, then the endotoxin plasmid gross extraction kit was applied to extract the plasmid. Each recombinant plasmid was amplified and purified for the subsequent experiments.

RNAi-mediated inhibition of ZNF139 in vitro. Experiments were divided into the blank control, siRNA-ZNF139 and negative control groups. Based on the transfection reagent instructions of the X-tremeGENE HP DNA transfection reagent (Roche, Basel, Switzerland), the recommended amount of plasmid $(0.8 \mu \mathrm{g} / \mu \mathrm{l})$ and the appropriate transfection reagents were added for cell transfection. Transfected cells $(\sim 200 \mu \mathrm{l})$ were seeded in each well of the 96-well plate and then incubated in $5 \% \mathrm{CO}_{2}$ at $37^{\circ} \mathrm{C}$ for $0,24,48$ and $72 \mathrm{~h}$ post-transfection. Approximately $20 \mu \mathrm{l}$ of MTT solution $(5 \mathrm{mg} / \mathrm{ml})$ was added into each well of the plate and incubated for another $4 \mathrm{~h}$. The supernatant was carefully discarded and an absorbent paper absorbed the residual liquid. A total of $150 \mu 1$ dimethyl sulfoxide (DMSO) was added to each well and agitated for $10 \mathrm{~min}$. The optical density (OD) of each well was measured at $570 \mathrm{~nm}$ to illustrate the cell growth curve.

RNAi-mediated inhibition of ZNF139 in vivo. The in vitro cultured SGC7901 human GC cells were collected and subcutaneously injected into nude mice. In total, $45 \mathrm{BALB} / \mathrm{C}$ male nude mice (age, 4 weeks; weight, 17-23 g) were used, including 18 mice for inter-mouse passage subcutaneous tumor transplantation and 27 mice for orthotopic tumor transplantation. The mice were obtained from the Fourth Hospital of Hebei Medical University Animal Center specific-pathogen-free animal laboratory (Shijiazhuan, China). Inter-mouse passage of the subcutaneous tumor was performed (Fig. 1B) and orthotopic transplantation was conducted on the sixth generation of subcutaneous tumor using the OB biological glue method (Fig. 1C) (6). The incision and growth of the abdominal orthotopic tumor graft were observed daily. Interference was conducted when the orthotopic tumor graft grew to $\geq 10 \mathrm{~mm}$ in diameter. The orthotopic grafted nude mice were randomly divided into the aforementioned three groups when the grafted tumor was developed.

The orthotopic xenografted nude mice were randomly divided into three groups ( $n=9$ per group). The grouping and intervention methods were as follows: In the blank control group, $50 \mu \mathrm{l}$ of saline was injected at multiple points at the tumor spot every other day. In the siRNA-ZNF139 group, $50 \mu 1$ of the intervention complex was administered by multipoint injection at the tumor spot every other day. The intervention complex included $20 \mu \mathrm{g}$ of the siRNA-ZNF139 plasmid and $20 \mu \mathrm{l}$ of the transfection reagent. The medium was added to a total volume of $50 \mu \mathrm{l}$. In the negative control group, $50 \mu \mathrm{l}$ of the intervention complex was administered by multipoint injection at the tumor spot every other day. The complex included $20 \mu \mathrm{g}$ of the negative-control plasmid and $20 \mu \mathrm{l}$ of the transfection reagent. The medium was added to a total volume of $50 \mu \mathrm{l}$.

During the intervention period following formation of the orthotopic tumor graft, the long and short diameters of the tumor were measured once every other day. The orthotopic tumor volume was also calculated with the following equation: $\mathrm{V}=\mathrm{ab}^{2} / 2$ (where $\mathrm{a}$ is the long diameter and $\mathrm{b}$ is the short diameter of the tumor). Two weeks after the intervention treatment, the nude mice were sacrificed using the cervical dislocation method. The in situ tumor was removed and the specimens were stored at $-80^{\circ} \mathrm{C}$ for future use. The experiment was performed in strict accordance with the Guide for the Care and Use of Laboratory Animals of the National Institutes of Health. The animal use protocol was reviewed and approved 
by the Institutional Animal Care and Use Committee of Hebei Medical University (permit no. 20060828001).

MTT assay. The SGC7901 human GC cells from different experimental groups and nude mice with in situ grafted tumors were prepared. Approximately $180 \mu \mathrm{l}$ of the cell suspension were seeded in each well of the 96-well plate. The three chemotherapeutic agents used were 5-fluorouracil (5-FU), cisplatin (CDDP) and mitomycin C (MMC). Each agent ( $20 \mu \mathrm{l})$ was added into each well of the 96 -well plate to a total volume of $200 \mu 1$ per well. The procedure was repeated five times. The final concentrations of the chemotherapeutic agents were $25 \mu \mathrm{g} / \mathrm{ml} 5-\mathrm{FU}, 4 \mu \mathrm{g} / \mathrm{ml} \mathrm{CDDP}$ and $4 \mu \mathrm{g} / \mathrm{ml}$ MMC. The plate was incubated in $5 \% \mathrm{CO}_{2}$ at $37^{\circ} \mathrm{C}$ for $48 \mathrm{~h}$. Following incubation, $20 \mu \mathrm{l}$ of MTT $(5 \mathrm{mg} / \mathrm{ml})$ was added to each well and incubated for another $4 \mathrm{~h}$. The supernatant was carefully discarded and the residual liquid was absorbed with an absorbent paper. Following addition of $150 \mu \mathrm{l}$ DMSO to each well and vortex mixing for $10 \mathrm{~min}$, the OD value of each well was measured at $570 \mathrm{~nm}$ with a microplate reader (Anthos 2010; Anthos Labtec Instruments GmBH, Wals-Siezenheim, Austria). The mean cell growth inhibitory rate (IR) was calculated as follows: $\mathrm{IR}=\left(1-\right.$ mean $\mathrm{OD}_{\text {drug treatment well }} /$ mean $\left.\mathrm{OD}_{\text {control well }}\right) \times 100 \%$.

Preparation of protein samples. The Bradford method (7) was used to detect the protein sample concentrations. Protein samples from the two experimental groups $(\sim 50 \mu \mathrm{g})$ were collected and labelled with CyDye 5 and CyDye3. Approximately $50 \mu \mathrm{g}$ of the protein samples in the control and experimental groups were replenished. Active hydration was conducted at $50 \mathrm{~V}$ for $10 \mathrm{~h}$ and then at $500 \mathrm{~V}$ for $1 \mathrm{~h}, 1,000 \mathrm{~V}$ for $1 \mathrm{~h}, 8,000 \mathrm{~V}$ for $1 \mathrm{~h}$ and $8,000 \mathrm{~V}$ for $3 \mathrm{~h}$. The final voltage increased from 10,000 to $65,000 \mathrm{~V}$. The strips were transferred to $10 \mathrm{ml}$ of balance solution A $[75 \mathrm{mmol} / 1$ Tris- $\mathrm{HCl}(\mathrm{pH} 8.8)$, $6 \mathrm{~mol} / \mathrm{l}$ urea, 30\% glycerol, 2\% sodium dodecyl sulfate (SDS) and $1 \%$ disulfide dithiothreitol (DTT); Sigma-Aldrich, St Louis, $\mathrm{MO}$, USA] for $15 \mathrm{~min}$ and then to balance solution B $(2.5 \%$ indole-3-acetic acid was used to replace the $1 \%$ DTT in solution A) for another $15 \mathrm{~min}$. The $12.5 \% \mathrm{SDS}$-polyacrylamide gel (PAGE) electrophoresis was performed at $50 \mathrm{~V}$ for $2 \mathrm{~h}$ and then at $120 \mathrm{~V}$ until the frontier of bromophenol blue was $\sim 1 \mathrm{~cm}$ from the lower end of the gel. Scanning was performed by Typhoon 9400 (GE Healthcare, Little Chalfont, UK), and image analysis was conducted by DeCyder difference analysis software (GE Healthcare).

Liquid chromatography-mass spectrometry (LC-MS) and database query. Following Deep Purple staining, an Ettan Spot Picker (GE Healthcare) was used to dig the spot. LTQXL ${ }^{\mathrm{TM}}$ (Thermo Fisher Scientific, Waltham, MA, USA) was used for the mass identification of the samples following in-gel digestion. BioWorks 3.3.1 software (Thermo Fisher Scientific) was used for database searching. The FASTA-form protein database was obtained from the National Center for Biotechnology Information human protein sequence database. Cysteine reductive alkylation and methionine oxidation were set as the fixed and variable modifiers, respectively.

Western blot analysis. Approximately $50 \mu \mathrm{g}$ of the prepared protein sample from each experimental group was separated by SDS-PAGE electrophoresis. The gel was sliced and transferred onto the polyvinylidene fluoride membrane, which was initially treated with the closure solution at room temperature and agitated for $1 \mathrm{~h}$. The primary antibodies [rabbit anti-human polyclonal antibodies against ZNF139 (cat. no. ab126124), pyridoxal kinase (PDXK; cat. no. ab38208), annexin A2 (ANXA2; cat. no. ab41803) and fascin (cat. no. ab183891), and mouse anti-human monoclonal antibody against $\beta$-actin (cat. no. ab6276); Abcam, Cambridge, $\mathrm{UK}$ ] were added and then incubated at $4^{\circ} \mathrm{C}$ overnight. The membrane was washed, followed by the addition of horseradish peroxidase enzyme-labelled goat anti-rabbit IgG secondary antibody (cat. no. ab6721; Abcam) and incubated at room temperature for $1 \mathrm{~h}$. The membrane was washed again and stained by chemiluminescence. The Bio-Rad Image Analysis system (Bio-Rad Laboratories, Inc., Hercules, CA, USA) was used with $\beta$-actin as the internal reference. The experiment was performed thrice.

Statistical analysis. Measured data are expressed as the mean \pm standard deviation and analysed by SPSS 13.0 statistical software (SPSS, Inc., Chicago, IL, USA). The intergroup comparison was conducted by analysis of variance.

\section{Results}

RNAi-mediated inhibition of ZNF139 in vitro. The growth of the SGC7901 human GC cells of the siRNA-ZNF139 group slowed down significantly compared with that of the control and negative control groups $(\mathrm{P}<0.05)$ (Fig. 2A).

RNAi-mediated inhibition of ZNF139 in vivo. The size of the human in situ grafted GC tumor of the siRNA-ZNF139 group was significantly decreased compared with that of the control and negative control groups $(\mathrm{P}<0.05)$ (Fig. 2B).

Chemosensitivity changes. The average IR of the cells of the siRNA-ZNF139 group was significantly higher $(\mathrm{P}<0.05)$ compared with that of the control and negative control groups (Table I).

$2 D-D I G E$. According to the 2D-DIGE spectrum of the SGC7901 human GC cells and in situ grafted GC tumors, the protein points were $1,958 \pm 67$ with $90.5 \%$ matching rate and $5,227 \pm 59$ with $88.7 \%$ matching rate, respectively. DeCyder difference analysis software was used to select the eight and six distinct protein points in the SGC7901 human GC cells and in situ grafted GC tumors, respectively. The experiment was performed thrice.

Identification of LC-MS. The Ettan spot picker was used to assess the different protein spots. Following in-gel digestion, LC-MS was used for the analysis. Among the different proteins of the SGC7901 human GC cells, six were identified by LC-MS, namely PDXK, desmin, nuclear phosphoprotein (NPM), heat-shock protein 70 (HSP70), ANXA2 and fascin. In the siRNA-ZNF139-transfected SGC7901 cells, PDXK, desmin and NPM were upregulated, whereas HSP70, ANXA2 and fascin were downregulated. Five proteins were identified by LC-MS among the different proteins in the in situ grafted 
Table I. Alterations in cell chemosensitivity following RNA interference.

\begin{tabular}{lccccccc}
\hline \multirow{2}{*}{$\begin{array}{l}\text { Experimental } \\
\text { groups }\end{array}$} & \multicolumn{3}{c}{ SGC7901 human GC cells } & & \multicolumn{3}{c}{ Human in situ grafted GC cells } \\
\cline { 2 - 3 } & 5 -FU & CDDP & MMC & & 5 -FU & CDDP & MMC \\
\hline Blank control & $29.18 \pm 3.01$ & $30.67 \pm 1.55$ & $19.58 \pm 2.17$ & & $31.25 \pm 2.86$ & $32.64 \pm 1.69$ & $21.29 \pm 2.23$ \\
siRNA-ZNF139 & $40.08 \pm 1.97^{\mathrm{a}}$ & $39.76 \pm 1.24^{\mathrm{a}}$ & $33.91 \pm 1.39^{\mathrm{a}}$ & & $42.28 \pm 2.13^{\mathrm{a}}$ & $41.94 \pm 1.53^{\mathrm{a}}$ & $35.70 \pm 1.66^{\mathrm{a}}$ \\
Negative control & $28.98 \pm 3.21$ & $30.50 \pm 1.84$ & $19.52 \pm 1.77$ & & $31.47 \pm 2.75$ & $32.48 \pm 1.91$ & $21.48 \pm 1.94$ \\
\hline
\end{tabular}

Mean cell inhibition rate $(\mathrm{IR})=\left(1-\right.$ mean $\mathrm{OD}_{\text {drug treatment well }} /$ mean $\left.\mathrm{OD}_{\text {control well }}\right) \times 100 \%$. Data are presented as the mean percentage \pm standard deviation. ${ }^{\mathrm{P}} \mathrm{P}<0.05$ compared with the blank control group. GC, gastric cancer; siRNA-ZNF139, small interfering RNA of zinc finger protein 139; OD, optical density; 5-FU, 5-fluorouracil; CDDP, cisplatin; MMC, mitomycin C.

Table II. Identification results of difference proteins.

\begin{tabular}{lllllrrr}
\hline Number & Proteins & Version & MW & Score & Coverage & pI & Expression \\
\hline 1 & PDXK & gil4505701 & 35079.90 & 30.14 & 11.20 & 5.75 & $\mathrm{U}$ \\
2 & Desmin & gil55749932 & 53503.23 & 30.17 & 6.60 & 5.21 & $\mathrm{U}$ \\
3 & NPM & gil10835063 & 32554.90 & 10.18 & 3.70 & 4.64 & $\mathrm{U}$ \\
4 & HSP70 & gil5123454 & 69995.16 & 30.16 & 4.70 & 5.47 & $\mathrm{D}$ \\
5 & ANXA2 & gil50845386 & 38579.82 & 40.23 & 2.90 & 7.57 & $\mathrm{D}$ \\
6 & Fascin & gil4507115 & 54496.09 & 10.21 & 2.80 & 6.84 & $\mathrm{D}$ \\
7 & ANXA1 & gil4502101 & 38690.00 & 60.20 & 17.30 & 6.57 & $\mathrm{U}$ \\
8 & hnRNP & gil55956919 & 35945.22 & 40.21 & 12.70 & 6.49 & $\mathrm{D}$ \\
\hline
\end{tabular}

MW, molecular weight; pI, isoelectric point; U, upregulated following ZNF139 inhibition; D, downregulated following ZNF139 inhibition; ZNF139, zinc finger protein 139; PDXK, pyridoxal kinase; ANXA2, annexin A2; NPM, nuclear phosphoprotein; HSP70, heat-shock protein 70; hnRNP, heterogenous nuclear ribonucleoprotein.
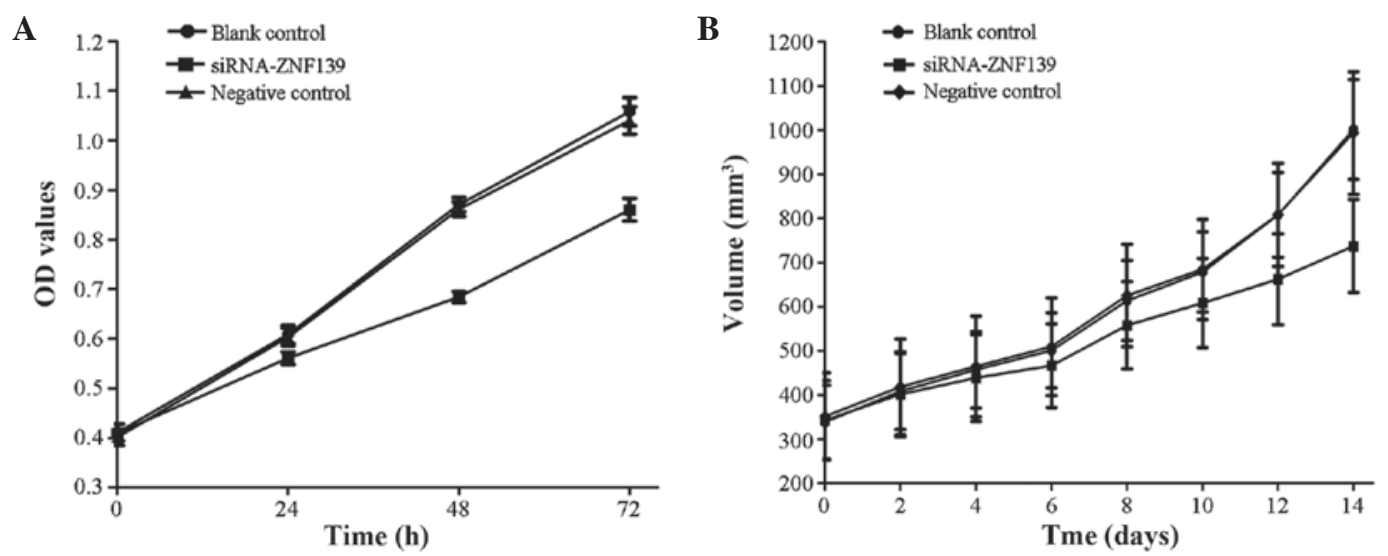

Figure 2. (A) Cell growth of SGC7901 human GC cells at different time points and (B) tumor size changes of human in situ grafted GC tumors following RNA interference. GC, gastric cancer; OD, optical density; siRNA-ZNF139, small interfering RNA of zinc finger protein 139.

human GC tumors, namely ANXA1, PDXK, fascin, ANXA2 and heterogeneous nuclear ribonucleoprotein (hnRNP). Following transfection, ANXA1 and PDXK were upregulated, whereas fascin, ANXA2 and hnRNP were downregulated (Table II and Fig. 3).

Western blot analysis. The expression of ZNF139 was significantly decreased in the siRNA-ZNF139 group compared with that in the control and negative control groups. PDXK expression was significantly increased, whereas the expression of ANXA2 and fascin was significantly decreased, in accordance with the proteomics results (Fig. 4).

\section{Discussion}

A previous study (8) demonstrated that siRNA may guide the RNA-induced silencing complex (RISC) to cut the homologous single-stranded mRNA and function as the primer to bind the 

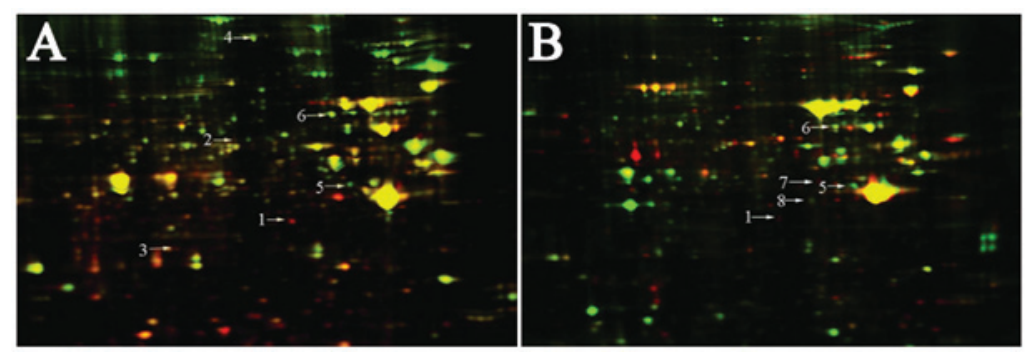

Figure 3. Two-dimensional difference gel electrophoresis spectrum of human gastric cancer (A) SGC7901 cell line and (B) in situ grafted tumor.

A

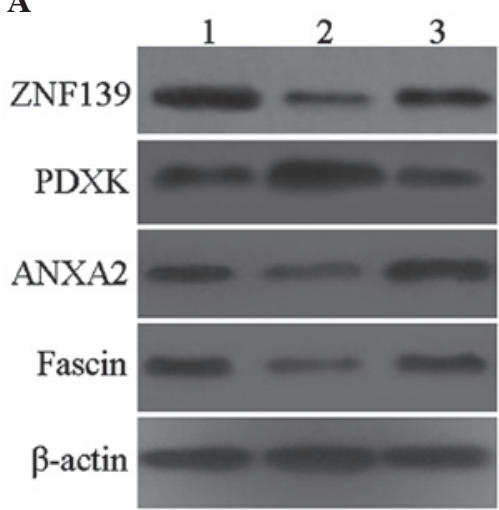

B

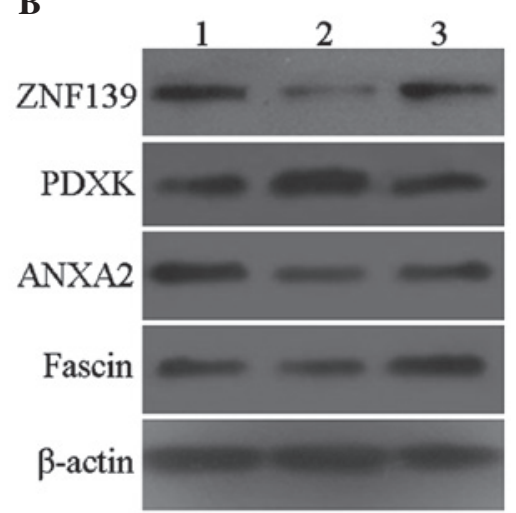

Figure 4. Western blot analysis of the expression of ZNF139, PDXK, ANXA2 and fascin in (A) SGC7901 cells and (B) transplanted tumors. 1, blank control group; 2, small interfering RNA-ZNF139 group; 3, negative control group. ZNF139, zinc finger protein 139; PDXK, pyridoxal kinase; ANXA2, annexin A2.

target RNA. A number of secondary dsRNAs are synthesized under RNA polymerase, and the newly synthesized dsRNA may be sliced by the Dicer enzyme to generate a large number of secondary siRNAs. Therefore, the RNAi effect increases further, resulting in the completion of the degradation of the target mRNA. The results of the present study demonstrated that siRNA-ZNF139 was able to inhibit the expression of ZNF139 and the growth of SGC7901 human GC cells and in situ grafted GC tumors in nude mice. These findings may provide the theoretical basis for the involvement of the ZNF139 gene in the occurrence, development and chemosensitivity of human GC.

The multidrug resistance (MDR) phenomenon of $\mathrm{GC}$ is a major problem that currently complicates clinical treatment, and one of the main reasons for the poor therapeutic effect of chemotherapy (9). MDR occurs when the tumor cells resist a specific anticancer drug and then exhibit cross-resistance to other, previously unencountered anticancer drugs, which may be structurally unrelated and have different targets and mechanisms of action (10). The fundamental solution for MDR is dependent on in-depth studies on its cause and underlying mechanism; such studies may provide a method for its complete reversion (11). Based on the present study, the application of siRNA to inhibit the expression of ZNF139 in SGC7901 human GC cells and in situ grafted GC tumors may increase the sensitivity of tumor cells to 5-FU, CDDP and MMC. The inhibition of ZNF139 expression in GC cells may improve the efficiency of chemotherapy in GC, thus providing a basis for future research on novel GC treatments.

Wasinger et al conducted a study applying proteomics (12); this technology has been widely used in various fields of biology due to its high sensitivity, accuracy and throughput. Franco et al (13) applied the combination of 2D-DIGE and LC-MS, and observed that the occurrence of GC was associated with the type of Helicobacter pylori strain. Xin et al (14) applied proteomics technology to compare the differences in SGC7901 human GC cells when cultured with and without methionine and observed that the lack of methionine may inhibit the proteins that are associated with the regulation of the apoptosis of GC cells. Yang et al (15) compared the proteome differences between the SGC7901 human GC cell line and a vincristine-resistant SGC7901 cell line, and identified a variety of MDR-related proteins. In the present study, siRNA-ZNF139 was applied to inhibit the expression levels of ZNF139 in SGC7901 human GC cells and in situ grafted GC tumors. Proteomics was used to identify the different proteins expressed prior to and following inhibition. Among the different identified proteins, PDXK, ANXA2 and fascin exhibited similar changes. Following transfection, PDXK was upregulated, whereas ANXA2 and fascin were downregulated. Therefore, ZNF139 may regulate the expression of PDXK, ANXA2 and fascin, thus participating in the occurrence, development and chemosensitivity of GC.

PDXK, a member of the ribose kinase superfamily, is widely present in biological organisms. PDXK is a key metabolic enzyme of vitamin B6 and catalyses its phosphorylation. The catalysed phosphorylation enables transfer into active pyridoxal-5'-phosphate (PLP), which has an important function in the synthesis and catabolism of amino acids. Matsubara et al (16) demonstrated that PLP is able to inhibit the proliferation of endothelial cells. Furthermore, Komatsu et al (17) demonstrated the inhibition of tumor cell growth by PLP. 
ANXs are mainly expressed inside the nucleus and cytoplasm and under the cell membrane; they are a class of structurally-related calcium-dependent phospholipid-binding proteins divided into five subfamilies, namely A, B, C, D and E. The A family of ANXs includes 13 members (A1-13) found inside vertebrate cells (18). ANXA2 was first observed inside Rous sarcoma virus-transformed chicken embryo fibroblasts, which are located in the human chromosome 15q21-q22. ANXA2 is composed of 12 introns and 13 exons, with 339 amino acids and a molecular weight of $36 \mathrm{kDa}(19)$. ANXA2 is mainly expressed inside endothelial cells, macrophages, mononuclear cells, nerve cells and certain tumor cells. This protein may be highly expressed in proliferating and transforming cells but its expression is low in terminally differentiated cells. It was recently demonstrated that ANXA2 is associated with various cytokines, including vascular endothelial growth factor (VEGF); this protein promotes VEGF expression on the tumor cell surface and the formation of new blood vessels, thus promoting the growth, invasion and metastasis of tumor cells (20). Zhang et al (21) demonstrated that ANXA2 is associated with MDR. Meng et al (22) applied proteomics technology to compare the parental human bladder cancer cell line (pumc-91) with the respective doxorubicin-resistant cell line (pumc-91/ADM) and observed that ANXA2 was highly expressed in pumc-91/ADM, which may indicate it is a resistance factor.

Fascin is an evolutionarily conserved cytoskeletal protein with a molecular weight of $55 \mathrm{kDa}$. The main function of fascin is to bind F-actin and assemble it in the parallel actin bundle. Fascin has an important function in cell migration, adhesion and exchange of intracellular information. During the occurrence and development of tumors, fascin protein expression increases, resulting in increased cell surface projections and decreased cell differentiation. Therefore, cell proliferation may be promoted, the actin cytoskeleton reconstructed and cell motility enhanced. Tumor cells exhibit enhanced invasiveness and migration ability, enabling them to penetrate the basement membrane and spread to the surrounding tissues (23).

In the present study, western blot analysis detected a significant increase in PDXK protein levels following siRNA-ZNF139 transfection. However, the expression levels of ANXA2 and fascin were significantly decreased. Therefore, ZNF139 may be involved in the occurrence, development and chemotherapeutic sensitivity of GC by promoting the expression of ANXA2 and inhibiting the expression of fascin and PDXK.

It was previously reported that the ZNF139 gene is closely associated with GC (4). With the aim to investigate novel GC treatments and governed by the principles of RNAi, we herein constructed an siRNA plasmid to the ZNF139 gene. Based on the analysis of the protein differences prior to and following siRNA-ZNF139 transfection, it was concluded that ZNF139 may promote the expression of ANXA2 and fascin, while suppressing the expression of PDXK. Therefore, ZNF139 may be significantly involved in the occurrence, development and chemosensitivity of GC. These findings are consistent with those of Komatsu et al (17), Zhang et al (21) and Meng et al (22). In conclusion, ZNF139 may inhibit the occurrence and development of GC, while increasing its sensitivity to chemotherapeutic agents, thus providing novel targets for the treatment of GC patients.

\section{References}

1. Are C, Rajaram S, Are M, et al: A review of global cancer burden: trends, challenges, strategies and a role for surgeons. J Surg Oncol 107: 221-226, 2013.

2. Bora RS, Gupta D, Mukkur TK and Saini KS: RNA interference therapeutics for cancer: challenges and opportunities (review). Mol Med Rep 6: 9-15, 2012.

3. Qu Y, Dang S and Hou P: Gene methylation in gastric cancer. Clin Chim Acta 424: 53-65, 2013.

4. Li Y, Tan BB, Zhao Q, Fan LQ, Liu Y and Wang D: Regulatory mechanism of ZNF139 in multi-drug resistance of gastric cancer cells. Mol Biol Rep 41: 3603-3610, 2014.

5. Parguiña AF and García A: Platelet proteomics in transfusion medicine: a reality with a challenging but promising future. Blood Transfus 10 (Suppl 2): 113-114, 2012.

6. Shi J, Wei PK, Zhang S, et al: OB glue paste technique for establishing nude mouse human gastric cancer orthotopic transplantation models. World J Gastroenterol 14: 4800-4804, 2008.

7. Kruger NJ: The Bradford method for protein quantitation. Methods Mol Biol 32: 9-15, 1994.

8. Castanotto D and Rossi JJ: The promises and pitfalls of RNA-interference-based therapeutics. Nature 457: 426-433, 2009.

9. Zhang X, Yashiro M, Qiu H, Nishii T, Matsuzaki T and Hirakawa K: Establishment and characterization of multidrug-resistant gastric cancer cell lines. Anticancer Res 30: 915-921, 2010.

10. Rocco A, Compare D, Liguori E, et al: MDR1-P-glycoprotein behaves as an oncofetal protein that promotes cell survival in gastric cancer cells. Lab Invest 92: 1407-1418, 2012.

11. Stein U, Fleuter C, Siegel F, et al: Impact of mutant $\beta$-catenin on ABCB1 expression and therapy response in colon cancer cells. $\mathrm{Br}$ J Cancer 106: 1395-1405, 2012.

12. Wasinger VC, Cordwell SJ, Cerpa-Poljak A, et al: Progress with gene-product mapping of the Mollicutes: Mycoplasma genitalium. Electrophoresis 16: 1090-1094, 1995.

13. Franco AT, Friedman DB, Nagy TA, et al: Delineation of a carcinogenic Helicobacter pylori proteome. Mol Cell Proteomics 8: 1947-1958, 2009.

14. Xin L, Cao WX, Fei XF, et al: Applying proteomic methodologies to analyze the effect of methionine restriction on proliferation of human gastric cancer SGC7901 cells. Clin Chim Acta 377: 206-212, 2007.

15. Yang YX, Xiao ZQ, Chen ZC, et al: Proteome analysis of multidrug resistance in vincristine-resistant human gastric cancer cell line SGC7901/VCR. Proteomics 6: 2009-2021, 2006.

16. Matsubara K, Matsumoto H, Mizushina Y, Lee JS and Kato N: Inhibitory effect of pyridoxal 5'-phosphate on endothelial cell proliferation, replicative DNA polymerase and DNA topoisomerase. Int J Mol Med 12: 51-55, 2003.

17. Komatsu S, Yanaka N, Matsubara K and Kato N: Antitumor effect of vitamin B6 and its mechanisms. Biochim Biophys Acta 1647: 127-130, 2003.

18. Gerke V and Moss SE: Annexins: from structure to function. Physiol Rev 82: 331-371, 2002.

19. Ozaki T and Sakiyama S: Molecular cloning of rat calpactin I heavy-chain cDNA whose expression is induced in v-src-transformed rat culture cell lines. Oncogene 8: 1707-1710, 1993.

20. Semov A, Moreno MJ, Onichtchenko A, Abulrob A, Ball M, Ekiel I, Pietrzynski G, Stanimirovic D and Alakhov V: Metastasis-associated protein S100A4 induces angiogenesis through interaction with annexin II and accelerated plasmin formation. J Biol Chem 280: 20833-20841, 2005.

21. Zhang F, Zhang L, Zhang B, et al: Anxa2 plays a critical role in enhanced invasiveness of the multidrug resistant human breast cancer cells. J Proteome Res 8: 5041-5047, 2009.

22. Meng Q, Lei T, Zhang M, Zhao J, Zhao XH and Zhang M: Identification of proteins differentially expressed in adriamycin-resistant (pumc-91/ADM) and parental (pumc-91) human bladder cancer cell lines by proteome analysis. J Cancer Res Clin Oncol 139: 509-519, 2013.

23. Hashimoto Y, Kim DJ and Adams JC: The roles of fascins in health and disease. J Pathol 224: 289-300, 2011. 